\title{
SPLIT FREE OSCILLATION AMPLITUDES FOR THE 1960 CHILEAN AND 1964 ALASKAN EARTHQUAKES
}

\author{
By Robert J. Geller and Seth Stein
}

\begin{abstract}
Splitting of the Earth's normal modes was observed for both the 1960 Chilean and 1964 Alaskan earthquakes. The strong peaks in the observed spectrum of each split multiplet correspond to singlets with much higher amplitudes than the others. Using theoretical results we have derived elsewhere (Stein and Geller, 1977a), we are able to predict this pattern. We show that the source mechanisms inferred for these earthquakes from surface waves are consistent with the observed pattern of relative spectral amplitudes of the split modes. However other mechanisms, such as a slow isotropic volume change, are also consistent with the split-mode amplitudes and are excluded only by additional data.
\end{abstract}

\section{INTRODUCTION}

Splitting of the Earth's normal modes was first observed by Benioff, Press, and Smith (1961), and Ness, Harrison, and Slichter (1961) for the Chilean earthquake. Pekeris, Alterman, and Jarosch (1961) and Backus and Gilbert (1961) calculated the frequency separation due to the Earth's rotation and obtained good agreement with the observed data. Pekeris et al. (1961) and Backus and Gilbert (1961) both calculated the relative amplitudes of each peak for some simple sources. However, the spectral amplitudes resulting from realistic models of the Alaskan and Chilean earthquakes as double-couple sources have not previously been calculated. In this paper, we use results we have previously developed (Stein and Geller, 1977a) to calculate the splitmode amplitudes for the Chilean and Alaskan earthquakes. Our predictions agree quite well with published observations of the split spectra.

\section{Theoretical Split-Mode Amplitudes}

The Earth's rotation (Pekeris et al., 1961 and Backus and Gilbert, 1961) and ellipticity (Dahlen, 1968) split the $2 l+1$ singlets belonging to the multiplet of angular order $l$ such that each one has a distinct eigenfrequency. The splitting is observable only for low angular order modes, for which the frequency separation of the singlets caused by rotation and ellipticity is greater than the broadening of spectral lines due to attenuation. For modes for which the splitting is observable, some singlets have much larger amplitudes than others in their multiplet. Thus the relative amplitudes of the split modes serve as an additional constraint in determining the character of earthquake source mechanisms at very long periods.

We have derived theoretical results (Stein and Geller, 1977a) that allow us to synthesize the split normal mode amplitudes excited by a realistic model of an earthquake source: a double couple of arbitrary orientation resulting from slip on a fault plane. (References to previous work are given in our earlier paper; we summarize our results here.) Our solution is obtained by transforming the spherical harmonic expansion of the excitation from the frame of reference of the source into geographic coordinates. We write the singlet amplitudes so that there are separate factors for source location (latitude and longitude), source depth, fault geometry (strike, dip, and slip direction), receiver location and the normalized energy of each mode. 
For the spheroidal modes and for a step-function dislocation with unit moment, the displacement of the normal mode with angular order $l$ and azimuthal order $m$ is given to zeroth order by

$$
\mathbf{U}^{S}(\mathbf{r}, t)=\mathbf{E}_{l m}(\mathbf{r}) \mathrm{e}^{i \omega_{l m} t}+\mathbf{E}_{l m}^{*}(\mathbf{r}) \mathrm{e}^{-i \omega_{l m} t}
$$

where $\omega_{l m}$ is the eigenfrequency of the mode. The overtone number, $n$, will be suppressed for convenience throughout this section.

The displacement spectral density of the $l m$ th mode is

$$
\mathbf{E}_{l m}(\mathbf{r})=D_{l m}\left\{y_{1}^{S}(r) \mathbf{S}_{l m}^{1}(\theta, \phi)+y_{3}^{S}(r) \mathbf{S}_{l m}^{2}(\theta, \phi)\right\}
$$

where $y_{1}{ }^{S}(r)$ and $y_{3}{ }^{s}(r)$ are the vertical and radial displacement eigenfunctions defined by Alterman et al. (1959) and implicitly depend on $l . \theta$ and $\phi$ are the colatitude and longitude in geographic coordinates. Dahlen (1968) shows that when rotation and ellipticity are the only perturbations, that the surficial eigenfunctions are, correct to zeroth order, the vector spherical harmonics in geographic coordinates, $\mathbf{S}_{l m}^{1}$ and $\mathbf{S}_{l m}^{2}$

$$
\begin{aligned}
& \mathbf{S}_{l m}^{1}(\theta, \phi)=\left(Y_{l m}(\theta, \phi), 0,0\right) \\
& \mathbf{S}_{l m}^{2}(\theta, \phi)=\left(0, \frac{\partial Y_{l m}(\theta, \phi)}{\partial \theta}, \frac{1}{\sin \theta} \frac{\partial Y_{l m}(\theta, \phi)}{\partial \phi}\right)
\end{aligned}
$$

where

$$
Y_{l m}(\theta, \phi)=(-1)^{m}\left[\frac{(l-m) !}{(l+m) !}\right]^{1 / 2} P_{l}^{m}(\cos \theta) e^{i m \phi} \quad(m \geq 0)
$$

and

$$
Y_{l m}(\theta, \phi)=(-1)^{m} Y_{l-m}^{*}(\theta, \phi) . \quad(m<0) .
$$

Here the associated Legendre polynomials are defined as

$$
P_{l}^{m}(x)=\left(1-x^{2}\right)^{m / 2} \frac{d^{m}}{d x^{m}} P_{l}(x)
$$

and

$$
P_{l}(x)=\frac{1}{2^{l} l !} \frac{d^{l}}{d x^{l}}\left(x^{2}-1\right)^{l}
$$

The eigenfunctions are the vector spherical harmonics in the geographic coordinates (about the north pole), since we model the Earth as being spherically symmetric. The only perturbations in the problem, rotation and ellipticity, are both symmetric about the rotation axis. To find the displacements, we need the excitation coefficients $D_{l m}$ for these vector spherical harmonics. These can be obtained from the excitation coefficients used for a nonrotating earth, which are computed in a frame of reference centered on the earthquake source (Figure 1). This has been done by Saito (1967).

To transform the excitation coefficients from the source coordinates to the geographic coordinates we make use of the rotation matrix elements (Brink and Satchler, 
1968) which transform spherical harmonics from one coordinate frame to another. Since the excitation coefficients can be regarded as the components of a vector whose bases are the vector spherical harmonics, they, too, can be transformed from one frame to the other.

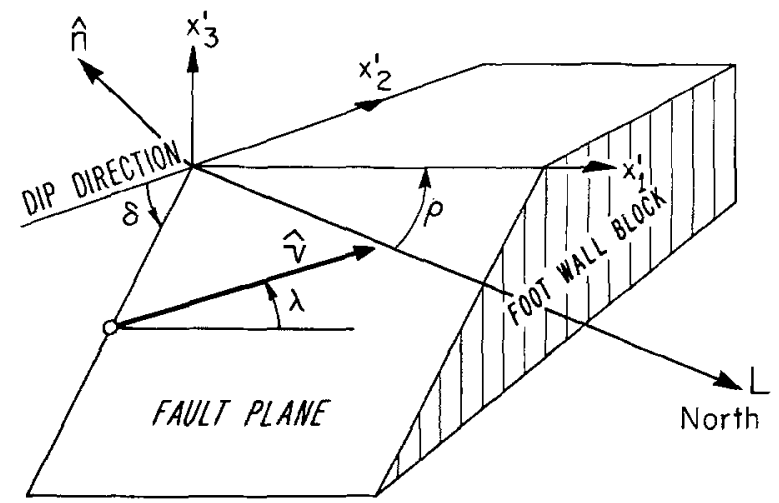

FIg. 1. Fault representation of Kanamori and Cipar (slightly modified). 0 is the slip vector, and gives the displacement of the hanging wall block. $\hat{\mathbf{n}}$ is the normal to the fault plane, and $I$ points north. The strike $p$ is measured counterclockwise from $L$. The dip angle $\delta$ is measured from the negative $X_{2}^{\prime}$ axis, and the slip angle $\lambda$ is measured in the fault plane counterclockwise from $\bar{X}_{1}^{\prime}$.

The rotation matrix elements are defined as

$$
\mathfrak{D}_{m k}^{l}(R)=\mathrm{e}^{-i(\alpha m+\gamma k)} d_{m k}^{l}(\beta)
$$

where (summing over all values of $t$ for which the factorials are non-negative),

$$
\begin{aligned}
d_{m k}^{l}(\beta)=\sum_{t}(-1)^{t}\left[\frac{(l+m) !(l-m) !(l+k) !(l-k) !]}{t !(l+m-t) !(l-k-t) !(t+k-m) !}\right]^{1 / 2} & \\
& \times[\sin (\beta / 2)]^{2 t+k-m}[\cos (\beta / 2)]^{2 l+m-k-2 t}
\end{aligned}
$$

The argument of these functions is the set of Euler angles $R=(\alpha, \beta, \gamma)$ that rotate the geographic coordinate system into the source coordinates. As shown in Figure 2, these angles are

$$
\begin{aligned}
& \alpha=\phi_{s} \\
& \beta=\theta_{s} \\
& \gamma=\pi+\rho
\end{aligned}
$$

where $\theta_{s}$ and $\phi_{s}$ are the epicentral coordinates and $\rho$ is the fault strike, measured counterclockwise from north. We describe all quantities in the source coordinates by primes $\left(X_{i}^{\prime}, D_{l m}^{\prime}\right)$ and their counterparts in the geographic coordinates without primes $\left(X_{i}, D_{l m}\right)$.

The excitation coefficients transform from the source frame to the geographic frame as

$$
D_{l m}=\sum_{k=-2}^{2} D_{m k}^{l}(R) D_{l k}^{\prime}
$$



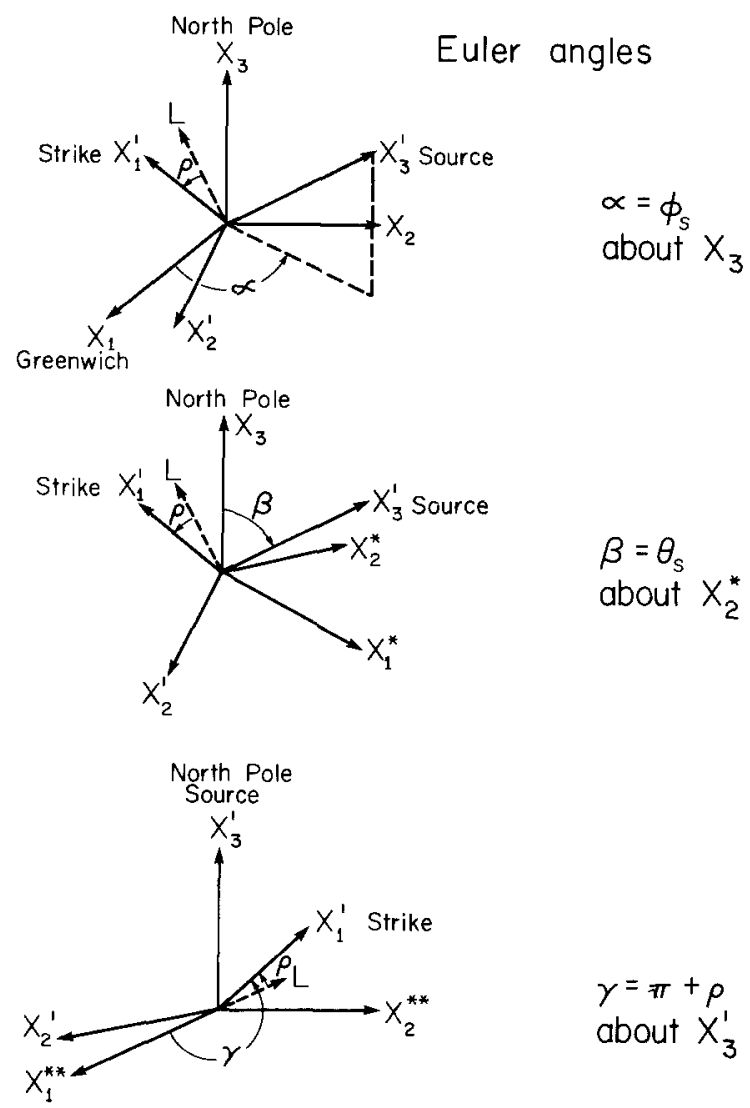

FIG. 2. Euler angles which rotate geographic $\left(X_{1}, X_{2}, X_{3}\right)$ axes into source $\left(X_{1}{ }^{\prime}, X_{2}{ }^{\prime}, X_{3}{ }^{\prime}\right)$ axes. All rotations are right-handed. The line $L$ points north in the $X_{1}^{\prime}-X_{2}^{\prime}$ plane.

The summation ranges over -2 to 2 , since for a point double couple only terms $D_{l m}^{\prime}$ with $|m| \leq 2$ are excited. Once the excitation is transformed to the geographic coordinates, all the terms from $-l$ to $l$ are excited. For such a source, with unit moment and a step-function time history, the source frame excitation coefficients can be expressed as

$$
\begin{aligned}
& D_{l 2}^{\prime}=K_{2} q_{2} C_{l 2} \\
& D_{l 1}^{\prime}=K_{1} q_{1} C_{l 1} \\
& D_{l 0}^{\prime}=K_{0} q_{0} C_{l 0} \\
& D_{l-1}^{\prime}=K_{1} q_{1}{ }^{*} C_{l-1} \\
& D_{l-2}^{\prime}=K_{2} q_{2}{ }^{*} C_{l-2} .
\end{aligned}
$$

The $C_{l m}$ are normalization constants

$$
\begin{array}{ll}
C_{l m}=\left[\frac{(l+m) !}{(l-m) !}\right]^{1 / 2}(-1)^{m} & \text { for } m \geq 0 \\
C_{l m}=C_{l-m}(-1)^{m} & \text { for } m<0 .
\end{array}
$$


The fault geometry defines the factors $q_{m}$, the radiation pattern coefficients,

$$
\begin{aligned}
& q_{0}=\frac{1}{2} \sin \lambda \sin \delta \cos \delta \\
& q_{1}=\frac{1}{4}(-\cos \lambda \cos \delta+i \sin \lambda \cos 2 \delta) \\
& q_{2}=\frac{1}{4}(-\sin \lambda \cos \delta \sin \delta-i \cos \lambda \sin \delta) .
\end{aligned}
$$

Here $\delta$ is the fault dip angle and $\lambda$ is the slip angle, measured counterclockwise from the strike as shown in Figure 1.

The source amplitude factors $K_{0}, K_{1}$ and $K_{2}$ were defined by Kanamori and Cipar (1974) to simplify the results of Saito (1967).

$$
\begin{aligned}
& \begin{array}{l}
K_{0}=\left(\frac{2 l+1}{4 \pi \omega l^{2}\left(I_{1}^{s}+L^{2} I_{2}^{s}\right) r_{s}}\right)\left(\frac{2\left(3 \lambda_{s}+2 \mu_{s}\right)}{\lambda_{s}+2 \mu_{s}}\right) \\
\quad \times\left(y_{1}^{s}\left(r_{s}\right)-\frac{r_{s}}{3 \lambda_{s}+2 \mu_{s}} y_{2}^{s}\left(r_{s}\right)-\frac{L^{2}}{2} y_{3}{ }^{s}\left(r_{s}\right)\right)
\end{array} \\
& K_{1}=\frac{2 l+1}{4 \pi \omega l^{2}\left(I_{1}^{s}+L^{2} I_{2}^{s}\right)} \frac{y_{4}^{s}\left(r_{s}\right)}{\mu_{s}} \\
& K_{2}=\frac{2 l+1}{4 \pi \omega l^{2}\left(I_{1}^{s}+L^{2} I_{2}^{s}\right)} \frac{y_{3}{ }^{s}\left(r_{s}\right)}{r_{s}} .
\end{aligned}
$$

$\lambda_{s}$ and $\mu_{s}$ are the values of the elastic moduli at the source depth, $r_{s}$, and $y_{i}\left(r_{s}\right)$ are the values of the eigenfunctions. $I_{1} s$ and $I_{2} s$ are energy integrals defined by Saito, $\omega l$ is the unperturbed eigenfrequency, and $L^{2}=l(l+1)$.

A similar analysis may be performed for an isotropic (purely explosive or compressional) source. For such a source, with step-function time history and a unit moment (for each of the three dipoles), equations (1) to (10) are unchanged. The only nonzero source frame excitation coefficient is the $m=0$ term (which yields an isotropic radiation pattern). Thus equation (11) becomes

$$
D_{l 0}^{\prime}=K_{0}^{\prime} C_{l 0}
$$

where the source amplitude factor $K_{0}{ }^{\prime}$ is adapted from Takeuchi and Saito (1972)

$$
K_{0}{ }^{\prime}=\frac{2 l+1}{4 \pi \omega_{l}{ }^{2}\left(I_{1}{ }^{s}+L^{2} I_{2}{ }^{s}\right)} \times\left[-\frac{4 \mu_{s}}{\left(\lambda_{s}+2 \mu_{s}\right)} \frac{y_{1}{ }^{s}\left(r_{s}\right)}{r_{s}}-\frac{y_{2}{ }^{s}\left(r_{s}\right)}{\left(\lambda_{s}+2 \mu_{s}\right)}+\frac{2 L^{2} \mu_{s} y_{3}{ }^{s}\left(r_{s}\right)}{\left(\lambda_{s}+2 \mu_{s}\right) r_{s}}\right] .
$$

Since the Chilean earthquake spectrum was observed on a strain meter, we compute the horizontal strain components

$$
\begin{aligned}
e_{\theta \theta} & =\frac{1}{r} \frac{\partial U_{\theta}}{\partial \theta}+\frac{U_{r}}{r} \\
e_{\phi \phi} & =\frac{1}{r \sin \theta} \frac{\partial U_{\phi}}{\partial \phi}+\frac{U_{\theta}}{r} \cot \theta+\frac{U_{r}}{r} \\
e_{\theta \phi} & =\frac{1}{2}\left[\frac{1}{r} \frac{\partial U_{\phi}}{\partial \theta}-\frac{U_{\phi} \cot \theta}{r}+\frac{1}{r \sin \theta} \frac{\partial U_{\theta}}{\partial \phi}\right] .
\end{aligned}
$$


The strains are given in geographic coordinates, so the strain in a strain rod of arbitrary orientation is

$$
e_{\text {rod }}=e_{\theta \theta} \cos ^{2} \gamma+2 e_{\theta \phi} \cos \gamma \sin \gamma+e_{\phi \phi} \sin ^{2} \gamma
$$

where $\gamma$ is the strain rod orientation measured counterclockwise from north.

\section{OBSERVEd Spectra}

There are very few reliable observations of split spectra. Not only are great lengths of record needed to resolve splitting, but, because attenuation broadens the peaks, even a very long record length does not allow resolution of splitting for any but the lowest angular order modes. This problem is discussed in Benioff et al. (1961).

Here we use the three best observations of split spectra, two for the Chilean earthquake $\left({ }_{0} S_{2}\right.$ and $\left.{ }_{0} S_{3}\right)$ and one for the Alaskan earthquake $\left({ }_{0} S_{2}\right)$. Using the theory sum-
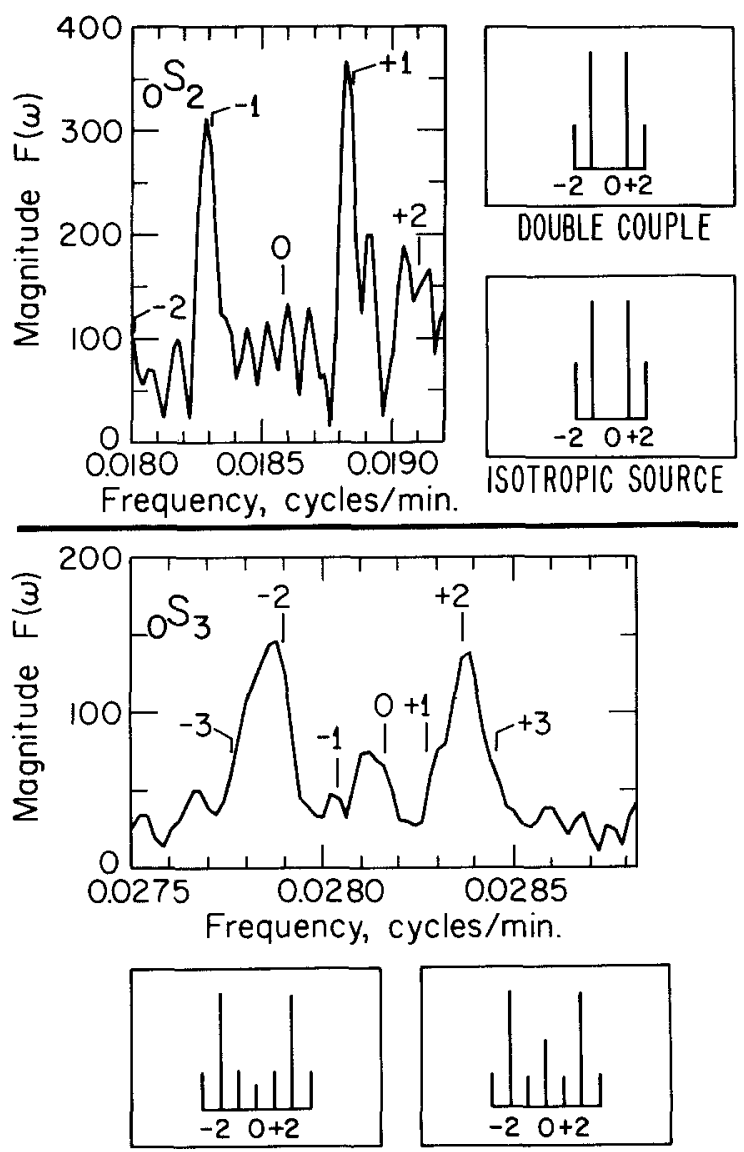

DOUBLE COUPLE

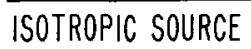

Frg. 3. Split spheroidal mode spectra for ${ }_{0} S_{2}\left(\right.$ top) and ${ }_{0} S_{3}$ (bottom) excited by the 1960 Chilean earthquake, as observed on a strainmeter at Isabella, California. The eigenfrequency separation is taken from Dahlen (1968), but the central frequency has been chosen to yield a best fit with the observed peaks. Synthetic relative spectra for an isotropic source and for the finite fault geometry of Kanamori and Cipar (1974) are given for each mode. The amplitudes are normalized and plotted with regular spacing. 
marized in the previous section, and fault geometries determined from the study of long-period surface waves, we compute synthetic spectra. We also compute synthetic spectra for an isotropic source, and compare the two sets of theoretical spectra to observations. Our synthetic relative spectral amplitudes are in excellent agreement with the data.

Figure 3 (from Benioff et al., 1961) shows the spectra of the spheroidal multiplets ${ }_{0} S_{2}$ (top) and $S_{3}$ (bottom) excited by the Chilean earthquake, as observed on a strainmeter (striking $38.4^{\circ} \mathrm{W}$ of $\mathrm{N}$ ) at Isabella, California. Dahlen's (1968) splitting parameters are used to identify the azimuthal order numbers of the peaks in Figure 3 and the center frequency is adjusted to give the best fit. The singlet pair with $m=$ \pm 1 has much larger amplitudes than the rest of the ${ }_{0} S_{2}$ multiplet and, similarly, ${ }_{0} S_{3} \pm 2$ stands out from its multiplet. (The results of a later analysis by Smith (1961) of the spectrum differed somewhat but did not alter the basic conclusion that ${ }_{0} S_{2} \pm 1$ and ${ }_{0} S_{3} \pm^{2}$ had much larger amplitudes than the other singlets of their multiplets.)

As well as the observed spectra, we show our synthetic relative spectral amplitudes computed for the finite fault geometry determined by Kanamori and Cipar (1974) from long-period surface-wave studies $\left(\rho=350^{\circ}, \lambda=90^{\circ}, \delta=10^{\circ}, \theta_{s}=128^{\circ}, \phi_{s}\right.$ $\left.=286.5^{\circ}, L=800 \mathrm{~km}, V_{R}=3.5 \mathrm{~km} / \mathrm{sec}\right)$ and including a precursory slip $\left(t_{p}=\right.$ $900 \mathrm{sec}$ and $\tau_{0}=300 \mathrm{sec}$ ) inferred from a time-domain observation (Kanamori and Cipar, 1974) and from spectral holes (Kanamori and Anderson, 1975). We also show the relative spectra for an isotropic point source, a model which was used by Pekeris et al. (1961) for the Chilean earthquake. For both sources the spectral amplitudes do not depend on the precise frequency separation, so for convenience we plot our theoretical amplitudes with regular spacing, although the actual spacing is somewhat asymmetric. The amplitudes of the split modes are symmetric for a point source; interference effects may cause a slight asymmetry for a finite source. However, in this case the finiteness has only a negligible effect on the relative spectral amplitudes.

Figure 4 shows the spectrum from a gravity meter record (Slichter, 1967) of the Alaskan earthquake, after processing and filtering by Wiggins and Miller (1972).

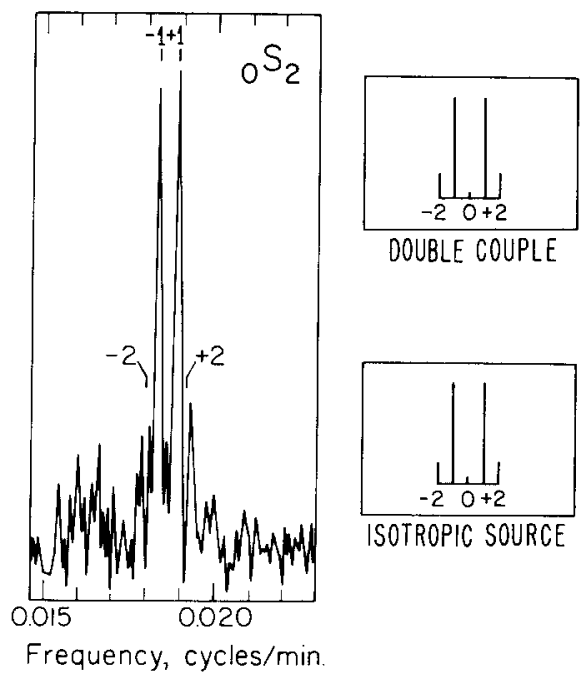

FIG. 4. Split spheroidal mode spectra for ${ }_{0} S_{2}$ excited by the Alaskan earthquake as observed on a gravity meter at Los Angeles, California by Slichter (1967) after processing by Wiggins and Miller (1972). Details are as in Figure 3. The finite fault geometry of Kanamori (1970) is used for the double-couple source. 
In this spectrum the singlet pair ${ }_{0} S_{2}^{ \pm 1}$ has much greater amplitude than the rest of the multiplet. Below this figure we show synthetic spectra (which have the same relative amplitudes as the vertical displacements) for the finite fault geometry ( $\rho=$ $114^{\circ}, \lambda=90^{\circ}, \delta=20^{\circ}, \theta_{s}=29.9^{\circ}, \phi_{s}=212.4^{\circ}, L=500 \mathrm{~km}, V_{R}=3.5 \mathrm{~km} / \mathrm{sec}$ ) determined by Kanamori (1970) and for an isotropic source.

For both earthquakes, the double-couple mechanism derived from long-period surface waves yields relative spectra which fit the observations. The exact amplitudes of the lower amplitude peaks are somewhat uncertain, and so the primary consideration is the overall agreement of our theoretical spectra with the observed peaks. Our calculations demonstrate that the fault geometry determined from surface waves at periods of several hundred seconds is consistent with free oscillation data at a period of almost one hour. However, the relative spectra for the isotropic source are also consistent with the observations and in fact closely resemble the double-couple spectra.

\section{Discussion}

We have described this similarity in some detail (Stein and Geller, 1977a), but a briefer explanation will suffice here. In the frame of reference of the earthquake source, the radiation patterns of ${ }_{0} S_{2}$ and ${ }_{0} S_{3}$ for a double couple are nearly radially symmetric, because the radially symmetric term $\left(D_{l_{0}}^{\prime}=K_{0} C_{l 0} q_{0}\right)$ is much larger than the two-lobed $\left(D_{l \pm 1}^{\prime}\right)$ or four-lobed $\left(D_{l \pm 2}^{\prime}\right)$ terms. Thus, for these low-order spheroidal modes, the radiation pattern of an earthquake nearly always resembles that of an isotropic source, which is completely radially symmetric. This resemblance is maintained where the excitation is transformed into geographic coordinates to calculate the split-mode amplitudes. The relative spectra of an earthquake will differ significantly from those of an isotropic source only in special cases, e.g. a pure strikeslip fault.

The isotropic and double-couple sources can be resolved very simply from the amplitudes of low-order torsional modes, since the former would not excite any torsional oscillations. Torsional oscillations are clearly visible for both the Chilean (Benioff et al., 1961) and Alaskan (Smith, 1966) earthquakes. In addition, long-period Rayleigh-wave (spheroidal oscillations) and Love-wave (torsional oscillations) data are consistent with a double-couple source, but not an isotropic source, up to periods of several hundred seconds (Kanamori, 1970; Kanamori and Cipar, 1974). Therefore, the double-couple source seems preferable in explaining the very-long-period free oscillation spectra. It is, of course, possible that an isotropic source component may exist as well as the dominant double-couple source. This is a difficult issue to resolve. Further study seems warranted; the observation of splitting for higher spheroidal models and for torsional modes could be useful in determining the mechanisms of these earthquakes, and others as well, at long periods.

For example, the theoretical singlet amplitudes of double couples depend strongly on fault geometry for spheroidal modes with $l>4$ and in general differ from those of an isotropic source. Thus, if splitting of these modes could be observed, it would be possible to test whether the fault mechanism determined from surface waves is appropriate for much longer-period free oscillations. Furthermore, once the mechanism has been determined, the absolute spectral amplitudes may be used to determine the moment at these much longer periods.

An alternate approach, which appears promising, is to study time-domain records of these earthquakes, for the lowest angular order modes. We have shown (Stein and Geller, 1977b) that modes $\left({ }_{0} S_{4}-{ }_{0} S_{5}\right.$ and $\left.{ }_{0} T_{3}-{ }_{0} T_{4}\right)$ for which splitting is difficult to 
observe in the spectra show the effects of splitting in the time series. By computing synthetic seismograms for different fault geometries, we can obtain additional information about the source.

Kanamori (1977) has summarized evidence showing that the interplate slip rates inferred from long-period surface waves are considerably smaller than those determined from magnetic anomalies. In view of the observations (Kanamori, 1972; Shimazaki and Geller, 1977) that in several cases the earthquake moment was still increasing at periods of several hundred seconds, it seems desirable to use low-order free oscillations to determine the moment at periods of almost one hour. Such determinations would allow us to distinguish between earthquake deformation having a time constant of one hour and longer-period aseismic slip which might involve large portions of the lithosphere. For past great earthquakes (e.g., Chile and Alaska), poorly determined instrument calibrations and the small number of long-period records present difficulties in determining absolute spectral amplitudes. The new long-period network (Agnew et al., 1976) should allow much more reliable very-long-period moment determinations.

\section{ACKNOWLEDGMENTS}

We thank Hiroo Kanamori for his advice and assistance, and for allowing us to use his results prior to publication. Seth Stein was supported by a fellowship from the Fannie and John Hertz Foundation and this research was supported by the National Science Foundation under Grants EAR74-22489 and EAR76-14262.

\section{REFERENCES}

Agnew, D., J. Berger, R. Buland, W. Farrell and F. Gilbert (1976). International deployment of accelerometers: a network for very long period seismology, EOS, Trans. Am. Geophys. Union, $57,180-188$.

Alterman, Z., H. Jarosch, and C. L. Pekeris (1959). Oscillations of the earth, Proc. Roy. Soc. London, Ser. A 252, 80-95.

Backus, G. and F. Gilbert (1961). The rotational splitting of the free oscillations of the earth, Proc. Natl. Acad. Sci. U.S., 47, 362-371.

Benioff, H., F. Press, and S. Smith (1961). Excitation of the free oscillations of the earth by earthquakes, $J$. Geophys. Res. 65, 605-619.

Brink, D. M. and G. R. Satchler (1968). Angular Momentum, Clarendon Press, Oxford.

Dahlen, F. A. (1968). The normal modes of a rotating, elliptical earth, Geophys. J., 16, 329-367.

Kanamori, H. (1970). The Alaska earthquake of 1964: Radiation of long-period surface waves and source mechanism, $J$. Geophys. Res. 75, 5029-5040.

Kanamori, H. (1972). Mechanism of tsunami earthquakes, Phys. Earth Planet. Interiors 6, 346359.

Kanamori, H. (1977). Seismic and aseismic slip along subduction zones and their tectonic implications, Am. Geophys. Union Monograph (Ewing volume), (in press).

Kanamori, H. and D. L. Anderson (1975). Amplitude of the earth's free oscillations and longperiod characteristics of the earthquake source, J. Geophys. Res. 80, 1075-1078.

Kanamori, H. and J. J. Cipar (1974). Focal process of the great Chilean earthquake, May 22, 1960, Phys. Earth Planet. Interiors 9, 128-136.

Ness, N., J. Harrison, and L. Slichter (1961). Observations of the free oscillations of the earth, $J$. Geophys. Res. 66, 621-629.

Pekeris, C. L., Z. Alterman, and H. Jarosch (1961). Rotational multiplets in the spectrum of the earth, Phys. Rev. 122, 1692-1700.

Saito, M. (1967). Excitation of free oscillations and surface waves by a point source in a vertically heterogeneous earth, J. Geophys. Res., 72, 3689-3699.

Shimazaki, K. and R. J. Geller (1977). Source process of the Kurile Islands tsunami earthquake of June 10, 1975, to be presented at 1977 Spring Annual Meeting of American Geophysical Union.

Slichter, L. B. (1967). Spherical oscillations of the earth, Geophys. J., 14, 171-177. 
Smith, S. W. (1961). An investigation of the Earth's free oscillations, Ph.D. Thesis, California Institute of Technology.

Smith, S. W. (1966). Free oscillations excited by the Alaskan earthquake, J. Geophys. Res. 71, 1183-1193.

Stein, S. and R. J. Geller (1977a). Amplitudes of the split normal modes of a rotating, elliptical earth excited by a double couple, J. Phys. Earth (submitted).

Stein, S. and R. J. Geller (1977b). Time-domain observation and synthesis of split spheroidal and torsional free oscillations of the 1960 Chilean earthquake: preliminary results, Geophys. Res. Lett., (submitted).

Takeuchi, H. and M. Saito (1972). Seismic surface waves, Methods in Computational Physics 11, 217-295.

Wiggins, R. A. and S. P. Miller (1972). New noise-reduction technique applied to long-period oscillations from the Alaskan earthquake, Bull. Seism. Soc. Am.62, 471-479.

Seismological Laboratory

California Institute of Technology

Pasadena, California 91125

Division of Geological and

Planetary Sciences

Contribution No. 2824

Manuscript received December 7, 1976 